\title{
Article \\ Neutrophil-to-Lymphocyte Ratio and Early Variation of NLR to Predict In-Hospital Mortality and Severity in ED Patients with SARS-CoV-2 Infection
}

\author{
Laure Abensur Vuillaume ${ }^{1,+}+{ }^{D}$, Pierrick Le Borgne ${ }^{2,3, *,+} \mathbb{D}$, Karine Alamé ${ }^{2, \dagger}$, François Lefebvre ${ }^{4}$, Lise Bérard ${ }^{5,+}$, \\ Nicolas Delmas ${ }^{6}$, Lauriane Cipolat ${ }^{1}$, Stéphane Gennai ${ }^{7}$, Pascal Bilbault ${ }^{2,3,+} \mathbb{D}$, Charles-Eric Lavoignet ${ }^{8, t}$ \\ and on behalf of the CREMS Network (Clinical Research in Emergency Medicine and Sepsis) ${ }^{\dagger}$
}

check for updates

Citation: Abensur Vuillaume, L.; Le Borgne, P.; Alamé, K.; Lefebvre, F.; Bérard, L.; Delmas, N.; Cipolat, L.; Gennai, S.; Bilbault, P.; Lavoignet, C.-E. Neutrophil-to-Lymphocyte Ratio and Early Variation of NLR to Predict In-Hospital Mortality and Severity in ED Patients with SARS-CoV-2 Infection. J. Clin. Med. 2021, 10, 2563. https://doi.org/ $10.3390 / \mathrm{jcm} 10122563$

Academic Editors: Emanuele Nicastri and Jose Bordon

Received: 1 April 2021

Accepted: 4 June 2021

Published: 9 June 2021

Publisher's Note: MDPI stays neutral with regard to jurisdictional claims in published maps and institutional affiliations.

Copyright: (c) 2021 by the authors. Licensee MDPI, Basel, Switzerland. This article is an open access article distributed under the terms and conditions of the Creative Commons Attribution (CC BY) license (https:// creativecommons.org/licenses/by/ $4.0 /)$.
1 Emergency Department, Regional Hospital of Metz-Thionville, 57000 Metz, France; 1.abensurvuillaume@chr-metz-thionville.fr (L.A.V.); 1.cipolat@chr-metz-thionville.fr (L.C.)

2 Emergency Department, Hôpitaux Universitaires de Strasbourg, 67000 Strasbourg, France; karine.alame@chru-strasbourg.fr (K.A.); pascal.bilbault@chru-strasbourg.fr (P.B.)

3 INSERM (French National Institute of Health and Medical Research), UMR 1260, Regenerative NanoMedicine (RNM), Fédération de Médecine Translationnelle (FMTS), University of Strasbourg, 67000 Strasbourg, France

4 Department of Public Health, University Hospital of Strasbourg, 67000 Strasbourg, France; francois.lefebvre@chru-strasbourg.fr

5 Emergency Department, Haguenau Hospital, 67500 Haguenau, France; lise.berard@ch-haguenau.fr

6 Emergency Department, Colmar Hospital, 68000 Colmar, France; nicolas.delmas@ch-colmar.fr

7 Emergency Department, Reims University Hospital, 51000 Reims, France; sgennai@chu-reims.fr

8 Emergency Department, Hôpital Nord Franche Comté, 90400 Trévenans, France; charles-eric.lavoignet@hnfc.fr

* Correspondence: pierrick.le-borgne@chru-strasbourg.fr; Tel.: +33-(0)-388-128-690; Fax: +33-(0)-388-128-100

+ Member of the CREMS Network (Clinical Research in Emergency Medicine and Sepsis), 67201 Wolfisheim, France.

Abstract: (1) Introduction: The neutrophil-to lymphocyte ratio is valued as a predictive marker in several inflammatory diseases. For example, an increasing NLR is a risk factor of mortality in sepsis. It also appears to be helpful in other settings such as cancer. The aim of our work was to study the prognostic value of NLR for disease severity and mortality in patients infected with SARS-CoV-2 upon their admission to the Emergency Department (ED) and its early variation ( $\triangle N L R)$ in the first $24 \mathrm{~h}$ of management (H-24). (2) Methods: Between 1 March and 30 April 2020, we conducted a multicenter and retrospective cohort study of patients with moderate or severe coronavirus disease 19 (COVID-19), who were all hospitalized after presenting to the ED. (3) Results: A total of 1035 patients were included in our study. Factors associated with infection severity were C-reactive protein level (OR: 1.007, CI 95\%: [1.005-1.010], $p<0.001$ ), NLR at H-24 (OR: 1.117, CI 95\%: [1.060-1.176], $p<0.001$ ), and $\triangle$ NLR (OR: 1.877, CI 95\%: [1.160-3.036], $p$ : 0.01). The best threshold of $\triangle$ NLR to predict the severity of infection was 0.222 (sensitivity $56.1 \%$, specificity $68.3 \%$ ). In multivariate analysis, the only biochemical factor significantly associated with mortality was again $\triangle$ NLR (OR: 2.142, CI 95\%: ([1.132-4.056], $p: 0.019$ ). The best threshold of $\Delta$ NLR to predict mortality was 0.411 (sensitivity $53.3 \%$; specificity $67.3 \%$ ). (4) Conclusion: The NLR and its early variation ( $\triangle$ NLR) could help physicians predict both severity and mortality associated with SARS-CoV-2 infection, hence contributing to optimized patient management (accurate triage and treatment).

Keywords: COVID-19; NLR; severity; mortality

\section{Introduction}

For over a year now, the world has been witnessing a global health crisis caused by an emerging coronavirus called severe acute respiratory syndrome coronavirus 2 (SARS-CoV2) which was first identified in Wuhan, China [1]. The Greater-East region of France was 
one of the pandemic's epicenters in Europe, where, as of mid-February 2021, this emerging virus resulted in nearly 7800 deaths and infected over 47,582 patients.

Although the pathophysiology of COVID-19 is not yet fully elucidated, it has been demonstrated that it correlates with a pro-inflammatory state. Studies have shown that this infection is associated with a dysregulation of the immune response, particularly inducing $\mathrm{T}$ lymphopenia with a frequent decrease in CD4+ $\mathrm{T}$ cells. This decrease in lymphocytes is more pronounced in severe SARS-CoV-2 infection due to the aggravated inflammatory response and production of a cytokine storm [2,3]. Thus, lymphopenia has often been regarded as the reference biomarker to judge the severity of COVID-19 [4]. This infectious disease, like other inflammatory states, is also followed by an increase in neutrophils [2]. However, neutrophilia, along with lymphopenia, is not specific for severity or mortality assessment in SARS-CoV-2 infection [5,6]. The NLR appears to be a good reflection of the systemic inflammatory response to a pathogen and may be more useful than lymphocytes or neutrophils alone in assessing the prognosis of patients with COVID-19 [7-10].

An increasing NLR is a risk factor of mortality in sepsis, where it can be a useful prognostic biomarker in critically ill patients [11,12]. This predictive marker appears to be helpful in other settings such as cancer [13]. Moreover, in emergency care, NLR appears to be a greater predictor of bacteriemia compared to other routine parameters such as Creactive protein (CRP) level and lymphocyte and neutrophil counts [14]. Recently, several studies have reported that patients with severe COVID-19 disease had a higher NLR value compared to patients with moderate disease [15,16]. In a recent meta-analysis of 13 studies involving 1579 patients, Li et al. [17] reported that NLR had sensitivity and specificity values of, respectively, $78 \%$ and $85 \%$ for disease severity and sensitivity and specificity values of $83 \%$ each for disease mortality. Hence, NLR could be fully integrated as a severity and mortality predictor in the management of infected patients both in the Emergency Department (ED) and in the subsequent hospital stay. However, the use of NLR variation, especially in the early stages of patient management, does not seem to have been subjected to study before.

Our aim was to investigate the prognostic value of NLR for disease severity and mortality in patients infected with SARS-CoV-2, upon their admission to the ED and in the first $24 \mathrm{~h}$ of management (H-24), excluding those who might have other confounding factors affecting their blood counts.

\section{Methods}

\subsection{Study Population and Settings}

We conducted a retrospective multicentric study in six ED of the Northeast region of France. We led our study in two university hospitals (CHRU of Strasbourg and CHU of Reims) and four general hospitals (Colmar Hospital, Nord Franche-Comté Hospital, Metz-Thionville Hospital and Haguenau Hospital). These hospital centers, along with the entire Greater-East region of France, constituted one of the outbreak's epicenters in Europe during the first wave.

We included all adult patients who were hospitalized for COVID-19 after presenting to the ED between 1 March and 30 April 2020. All patients included in our study had a laboratory-confirmed diagnosis of COVID-19 by RT-PCR on nasopharyngeal swab. We excluded patients who had a non-confirmed diagnosis, those who received outpatient care, and those who received palliative therapy or limitation of therapeutic effort upon admission to the ED. Patients with a medical history or treatment that altered their blood counts and, therefore, their circulating lymphocytes or neutrophils (e.g., chemotherapy, immunosuppressive therapy, long- and short-term corticosteroid therapy, pre-admission antibiotic therapy, active cancer, or hematological malignancies) were also excluded from our study. 


\subsection{Data Collection}

We retrospectively queried patients' electronic medical records for epidemiological, clinical, and biochemical data and then standardized the results in a report file. We recorded symptom onset date along with patient's current treatment and medical history (including cardiovascular disease, diabetes, pre-existing renal failure, cancer, and hematological diseases). The primary endpoint was the prognostic value of NLR on in-hospital mortality. The secondary endpoint was its prognostic value on severity of disease, where severe disease was defined by patient admission to the ICU (patients under invasive mechanical ventilation), and moderate disease was defined by patient admission to conventional hospitalization units (most patients with oxygen therapy). Ambulatory patients were excluded. Obesity was defined by a body mass index superior to $30 \mathrm{~kg} / \mathrm{m}^{2}$. Functional autonomy was measured by the Knaus score. Standard biochemical parameters, such as levels of creatinine, CRP, total leukocytes and lymphocytes, were also collected. Lastly, we measured lymphocyte and neutrophil count and NLR early variation ( $\triangle N L R$ ), i.e., the difference between NLR values at $\mathrm{H}-24$ and upon admission to the ED.

\subsection{Ethics}

This study was approved by the local ethics committee of the University of Strasbourg in France (reference CE: 2020-39), which, in accordance with the French legislation, waived the need for informed consent of patients whose data were entirely retrospectively studied.

\subsection{Statistical Analysis}

The statistical analyses included a descriptive section and an analytical section. We performed a descriptive analysis of the categorical variables by providing the frequency of each value along with the cumulative frequency. We performed a descriptive analysis of the continuous variables by providing location parameters (mean, median, minimum, maximum, first and third quartiles) and dispersion parameters (standard deviation, variance, range, and interquartile range). Normality of the distributions was tested using a normality test, such as the Shapiro-Wilk or Kolmogorov-Smirnov test, and was assessed graphically using a normal quantile plot. Comparisons between categorical variables were performed using Chi-squared test or Fisher's exact test in case of expected values below 5 in any of the cells of the contingency table. Comparisons between continuous and categorical variables were assessed using the Student's t-test or Wilcoxon's test in case of heteroscedasticity or if the variable did not follow a normal distribution. Multivariate analyses were performed using all relevant variables. Multivariate analysis was performed using all variables obtained at admission or at H-24. Variables were selected using a backward stepwise method based on the Akaike Information Criterion to retain the relevant variables. The significance level was set at $5 \%$. All the statistical analyses were generated with R 4.0.2. The cut-off of $\triangle$ NLR was defined using the Youden's index. We created a validation cohort within our cohort by dividing it into two parts to confirm and justify our data (Supplementary Materials Tables S1-S4). Both cohorts were created using a random draw method.

\section{Results}

\subsection{Characteristics of the Study Population}

During the study period, a total of 49,326 patients were admitted to the ED of all six hospitals. Of these patients, 4470 had a laboratory-confirmed SARS-CoV-2 infection and, in fine, 1035 patients were included in our study (Figure 1).

Our cohort had a median age of 69 ((58-79)) years and was predominately male (58.8\%, CI 95\%: (55.8-61.8)). One-third of the study population was obese (34\%). In terms of medical history, over half of the patients $(56.7 \%)$ had high blood pressure, over a quarter of them $(26.7 \%)$ had a history of diabetes, and $23.2 \%$ of them presented pre-existing renal failure. At admission, the median NLR was significantly higher in the group presenting severe disease compared to that with moderate disease (5.2 ((3.2-8.7) versus $6.6(4.1-11.1)$, $p<0.001)$. Our findings were similar at H-24 (4.4 (2.7-7) versus 7.4 $(4.7-12.5), p<0.001)$. 
Thus, over half (58\%, CI 95\%: (52-64.3)) of the severely affected patients presented a positive $\triangle$ NLR. Principals clinical and biochemical patient characteristics are summarized in Table 1.

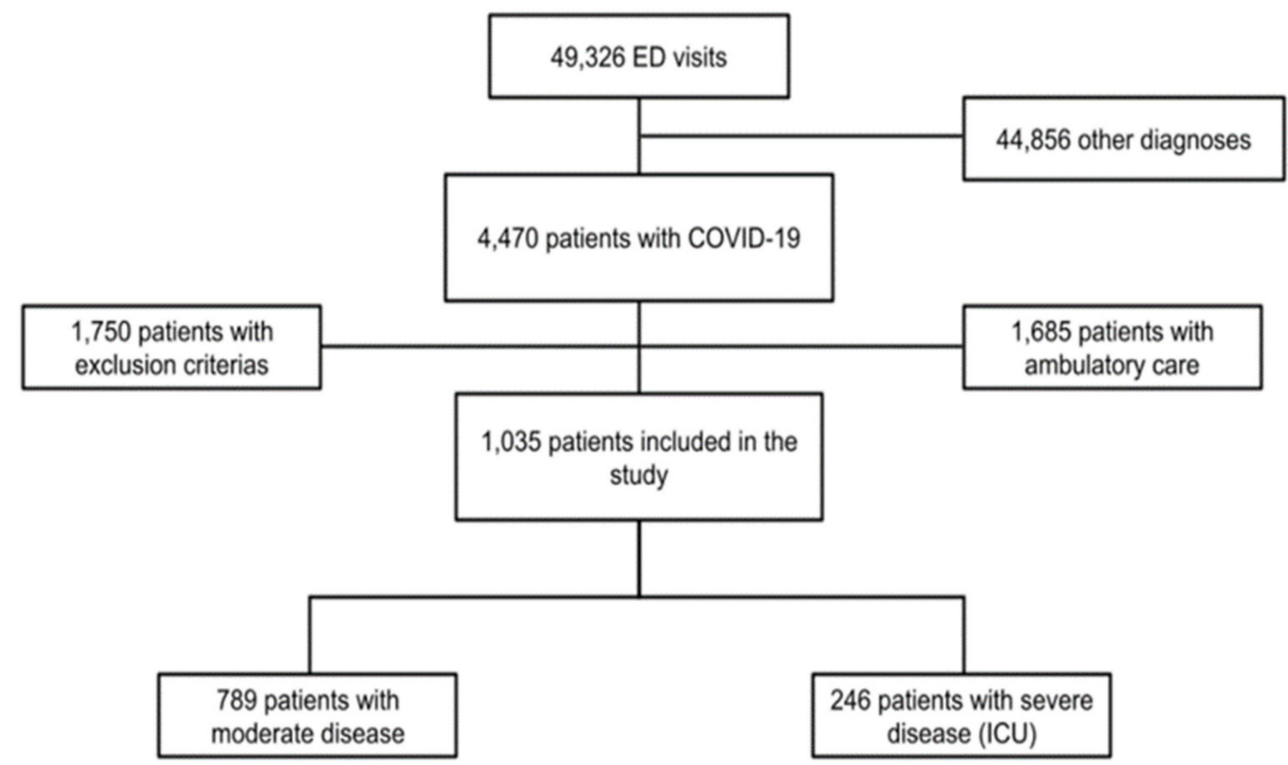

Figure 1. Flowchart of the study. Abbreviations: ED: Emergency Department, ICU: intensive care unit.

Table 1. Demographic, baseline, and laboratory characteristics of patients with COVID-19.

\begin{tabular}{|c|c|c|c|c|}
\hline & $\begin{array}{l}\text { All Patients } \\
n=1035\end{array}$ & $\begin{array}{c}\text { Moderate } \\
n=789\end{array}$ & $\begin{array}{l}\text { Severe } \\
n=246\end{array}$ & $p$ \\
\hline \multicolumn{5}{|l|}{ General Characteristics } \\
\hline Age (years) & $69(58-79)$ & $70(58-81)$ & $66(57.3-72)$ & $<0.001 *$ \\
\hline Gender male & $609(58.8)$ & $433(54.9)$ & $176(71.5)$ & $<0.001 *$ \\
\hline Obesity (BMI $\geq 30$ ) & $281(36.9)$ & $193(35.0)$ & $88(41.9)$ & 0.076 \\
\hline \multicolumn{5}{|l|}{ Chronic Medical Illness } \\
\hline Hypertension & $587(56.7)$ & $453(57.4)$ & $134(54.5)$ & 0.416 \\
\hline Diabetes mellitus & $275(26.7)$ & $202(25.6)$ & $73(26.6)$ & 0.207 \\
\hline CKD & $237(23.2)$ & $199(25.5)$ & $38(15.8)$ & $0.002 *$ \\
\hline Cardiovascular illness & $357(34.5)$ & $291(36.9)$ & $66(26.8)$ & 0.004 * \\
\hline Total Autonomy & 796 (77.2) & $569(72.4)$ & $227(92.7)$ & $<0.001 *$ \\
\hline Respiratory illness & $203(19.6)$ & $151(19.1)$ & $52(21.1)$ & 0.490 \\
\hline \multicolumn{5}{|l|}{ Laboratory Findings } \\
\hline $\mathrm{CRP}(\mathrm{mg} / \mathrm{L})$ & $81(39-142.3)$ & $68(33-128)$ & $124(76-192)$ & $<0.001 *$ \\
\hline Lymphocyte $\left(\times 10^{9} / \mathrm{L}\right)$ & $870(630-1200)$ & $900(640-1220)$ & $780(590-1122)$ & 0.003 * \\
\hline Lymphocyte H24 ( $\left.\times 10^{9} / \mathrm{L}\right)$ & $940(670-1300)$ & $1010(710-1360)$ & $800(570-1110)$ & $<0.001$ * \\
\hline Neutrophil $\left(\times 10^{9} / \mathrm{L}\right)$ & $4930(3430-6932)$ & $4730(3370-6620)$ & $5510(3760-8160)$ & $<0.001 *$ \\
\hline Neutrophil H24 (×109 /L) & $4680(3300-6765)$ & $4395(3005-6175)$ & $6010(4130-8210)$ & $<0.001$ * \\
\hline Admission NLR & $5.4(3.5-9.3)$ & $5.2(3.2-8.7)$ & $6.6(4.1-11.1)$ & $<0.001 *$ \\
\hline H-24 NLR & $5(3.1-8.2)$ & $4.4(2.7-7)$ & $7.4(4.7-12.5)$ & $<0.001$ * \\
\hline$\Delta \mathrm{NLR}>0(\%)$ & $334(41.3)$ & $211(35.3)$ & $123(58.0)$ & $<0.001$ * \\
\hline$\triangle \mathrm{NLR}$ & $-0.64(-2.88-1.29)$ & $-0.88(-3.09-0.79)$ & $0.48(-1.78-3.72)$ & $<0.001 *$ \\
\hline \multicolumn{5}{|l|}{ Outcome } \\
\hline Hospital stay (days) & $10(7-17.3)$ & $8(6-12)$ & $24(17-38)$ & $<0.001$ * \\
\hline Intra-hospital mortality & $139(13.6)$ & $82(10.4)$ & $57(24.1)$ & $<0.001 *$ \\
\hline
\end{tabular}

Data are expressed in median (Q1-Q3) $n(\%)$, where $n$ is the total number of patients with available data. ${ }^{*} p<0.05$. Abbreviations: BMI $=$ body mass index, $\mathrm{CKD}=$ Chronic kidney disease, $\mathrm{ED}=$ Emergency Department, $\mathrm{O} 2=$ oxygen,${ }^{\circ} \mathrm{C}=\mathrm{Celsius}$ degree, $\mathrm{CRP}=\mathrm{C}$ reactive protein, NLR= neutrophil to lymphocyte ratio, $\triangle N L R=$ difference between NLR (NLR H-24-NLR at admission). 


\subsection{Biochemichal Factors Associated with Severe COVID-19}

Of the total study population, 789 patients $(76.2 \%)$ had moderate disease, whereas $246(23.8 \%)$ had severe disease, which required ICU management. In multivariate analysis, the factors associated with the severity of the infection were CRP (OR: 1.007, CI 95\%: (1.005-1.010), $p<0.001)$, NLR at H-24 (OR: 1.117, CI 95\%: $(1.060-1.176, p<0.001)$, and $\triangle$ NLR (OR: 1.877, CI 95\%: (1.160-3.036); $p: 0.01)$. These values are summarized in Table 2.

Table 2. Biochemical factors associated with severe COVID-19 (admission to the ICU).

\begin{tabular}{cccccc}
\hline & & & \multicolumn{2}{c}{ Multivariate Analysis ** } \\
\hline CRP & All & Moderate & Severe & OR (95\% CI) & $p$-Value \\
\hline Admission & $81(39-142.3)$ & $68(33-128)$ & $124(76-92)$ & $1.007(1.005-1.010)$ & $<0.001 *$ \\
lymphocytes & $870(630-1200)$ & $900(640-1220)$ & $780(590-1122.5)$ & $1000(1.000-1.000)$ & 0.841 \\
Admission NLR & $5.4(3.5-9.3)$ & $5.2(3.2-8.7)$ & $6.6(4.1-11.1)$ & $0.971(0.940-1.004)$ & 0.082 \\
H-24 NLR & $5(3.1-8.2)$ & $4.4(2.7-7.0)$ & $7.4(4.7-12.5)$ & $1.117(1.060-1.176)$ & $<0.001 *$ \\
ANLR >0 (\%) & $334(41.3)$ & $211(35.3)$ & $123(58.0)$ & $1.877(1.160-3.036)$ & $0.010 *$ \\
\hline
\end{tabular}

Data are expressed in median (Q1-Q3) or $n(\%)$, where $n$ is the total number of patients with available data. ${ }^{*} p<0.05,{ }^{* *}$ model adjusted for gender, Body mass index, $\mathrm{C}$ reactive protein, Creatinine, Admission NLR, NLR H24 and $\triangle$ NLR. Abbreviations: OR $=$ odds ratio, $\mathrm{BMI}=$ body mass index, $\mathrm{CRP}=\mathrm{C}$ reactive protein, $\mathrm{NLR}=$ neutrophil to lymphocyte ratio, $\triangle \mathrm{NLR}=$ difference between NLR (NLR H-24-NLR at admission).

\subsection{Predictive Factors of Severe COVID-19}

We determined two ROC curves to predict the risk of disease severity. Regarding NLR at admission, the area under the curve (AUC) was 0.593 (CI 95\%: $(0.552-0.634), p<0.001$ ). The best cutoff for predicting the risk of infection severity was 6.883; it yielded a sensitivity of $48.3 \%$ (CI 95\% (41.9-54.8)) and a specificity of $65.6 \%$ (CI 95\% (62.2-69.0)). In multivariate analysis, if NLR was greater than 6.883, the OR was valued at 0.975 (CI 95\%: (0.947-1.005), p: 0.097).

Regarding $\triangle$ NLR, the AUC was 0.627 (CI 95\%: $(0.580-0.674), p<0.001)$. The best cutoff for predicting the risk of infection severity was 0.222 ; it provided a sensitivity of $56.1 \%$ (CI 95\% (49.2-62.9)) and a specificity of 68.3\% (CI 95\% (64.4-72.1)). In multivariate analysis, if $\triangle \mathrm{NLR}$ was greater than 0.022 , the OR was valued at 2.187 (CI 95\%: (1.348-3.547), $p:$ 0.002). These results are consistent with those of the validation cohort (Supplementary Materials Figure S1) and are presented in Figure 2.

\subsection{Biochemical Factors Associated with Mortality}

A total of 139 patients died during their hospital stay, representing $13.4 \%$ (CI 95\%: (11.4-15.5)) of our cohort. Upon admission to the ED and at H-24, the NLR values were associated with mortality in univariate analysis. However, the only biochemical parameter significantly associated with mortality in multivariate analysis was $\triangle$ NLR (OR: 2.142, CI 95\%: (1.132-4.056), p: 0.019). These results are summarized in Table 3. 


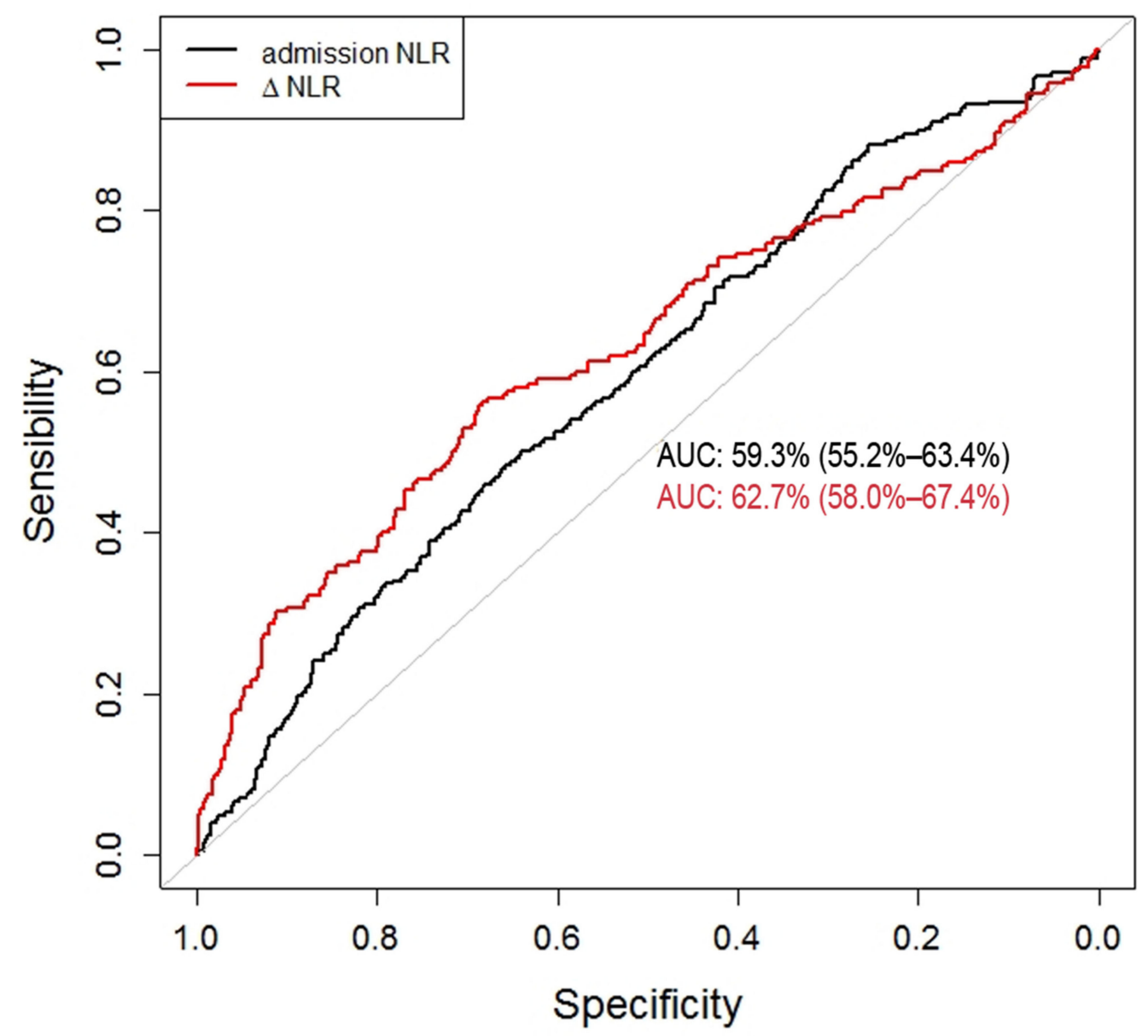

\begin{tabular}{ccccccc}
\hline \multirow{2}{*}{$\begin{array}{c}\text { To Predict } \\
\text { Critical Illness }\end{array}$} & Area under Curve & Cut-Off & Sensibility & Specificity & \multicolumn{2}{c}{$\begin{array}{c}\text { Multivariate Analyse with } \\
\text { Cut-Off** }\end{array}$} \\
\cline { 5 - 7 } & & & & OR (95\%CI) & $p$ \\
\hline Admission NLR & $0.593(0.552-0.634)$ & 6.883 & $48.3(41.9-54.8)$ & $65.7(62.2-69.0)$ & $0.975(0.947-1.005)$ & 0.097 \\
$\Delta$ NLR & $0.627(0.580-0.674)$ & 0.222 & $56.1(49.2-62.9)$ & $68.3(64.4-72.1)$ & $2.187(1.348-3.547)$ & $0.002 *$ \\
\hline
\end{tabular}

Data are expressed in median (Q1-Q3), * $p<0.005,{ }^{* *}$ model adjusted for Gender, Body mass index, $\mathrm{C}$ reactive protein, Creatinine, Admission NLR, NLR H24 and $\triangle$ NLR.

Abbreviations: $\mathrm{OR}=$ odds ratio, NLR: neutrophil lymphocyte ratio, $\triangle \mathrm{NLR}=$ difference between NLR (NLR H-24-NLR at admission).

Figure 2. Receiver operating characteristics (ROC) curve for NLR as a predictive factor of severe COVID-19.

Table 3. Biochemical and predictive factors of mortality in COVID-19 patients.

\begin{tabular}{|c|c|c|c|c|c|c|}
\hline & & & \multicolumn{2}{|c|}{ Univariate Analysis } & \multicolumn{2}{|c|}{ Multivariate Analysis ** } \\
\hline & Survivors & Non-Survivors & OR (95\% CI) & $p$-Value & OR $(95 \% \mathrm{CI})$ & $p$-Value \\
\hline CRP & $100.0(56-158)$ & $78.5(37-139)$ & $\begin{array}{c}1.003 \\
(1.001-1.005)\end{array}$ & $0.006^{*}$ & $\begin{array}{c}1.002 \\
(0.999-1.005)\end{array}$ & 0.276 \\
\hline $\begin{array}{l}\text { Admission } \\
\text { lymphocytes }\end{array}$ & $720(500-1000)$ & $890(650-1120)$ & $0.999(0.999-1)$ & 0.004 & $\begin{array}{c}1.000 \\
(0.999-1.001)\end{array}$ & 0.967 \\
\hline Admission NLR & $7.6(4.3-11.8)$ & $5.2(3.3-8.8)$ & $\begin{array}{c}1.023 \\
(1.006-1.042)\end{array}$ & 0.010 * & $\begin{array}{c}1.020 \\
(0.987-1.055)\end{array}$ & 0.232 \\
\hline H-24 NLR & $7.9(5.1-14.1)$ & $4.6(2.9-7.5)$ & $\begin{array}{c}1.059 \\
(1.035-1.084)\end{array}$ & $<0.001$ * & $\begin{array}{c}1.006 \\
(0.963-1.050)\end{array}$ & 0.798 \\
\hline$\Delta \mathrm{NLR}>0(\%)$ & $271(39.0)$ & $58(55.2)$ & $\begin{array}{c}1.931 \\
(1.277-2.920)\end{array}$ & $0.002 *$ & $\begin{array}{c}2.142 \\
(1.132-4.056)\end{array}$ & 0.019 * \\
\hline
\end{tabular}

Data are expressed in median (Q1-Q3) or $n(\%)$, where $n$ is the total number of patients with available data. ${ }^{*} p<0.05,{ }^{* *}$ model adjusted for Gender, Body mass index, $\mathrm{C}$ reactive protein, Creatinine, Admission NLR, NLR H24 and $\triangle$ NLR. Abbreviations: OR = odds ratio, $\mathrm{BMI}=$ body mass index, $\mathrm{CRP}=\mathrm{C}$ reactive protein, $\mathrm{NLR}=$ neutrophil to lymphocyte ratio, $\triangle \mathrm{NLR}=$ difference between NLR (NLR H-24-NLR at admission). 


\subsection{Predictive Factors of Mortality}

Regarding NLR at admission, the AUC was 0.621 (CI 95\%: (0.571-0.672)). The best NLR threshold for predicting the risk of death was 8.23; it yielded a sensitivity of $47.4 \%$ (CI 95\%: (38.8-56.2)) and a specificity of 71.9\% (CI 95\%: (68.8-74.9)). If NLR was greater than 8.23 , multivariate analysis was not found significant, with an OR valued at 1.025 (CI 95\%: (0.989-1.063), $p$ : 0.176). Regarding $\triangle \mathrm{NLR}$, the AUC was 0.576 (CI 95\% (0.509-0.643)). The best $\triangle$ NLR threshold for predicting the risk of death was 0.411 ; it yielded a sensitivity of 53.3\% (CI 95\% (43.3-63.1)) and a specificity of $67.3 \%$ (CI 95\% (63.7-70.8)). In multivariate analysis, if $\triangle \mathrm{NLR}$ was greater than 0.411 , then the OR was valued at 2.71 (CI $95 \%$ : $(1.404-5.245) ; p: 0.003)$. These results are presented in Figure 3 and are consistent with those of the validation cohort (Supplementary Materials Figure S2).

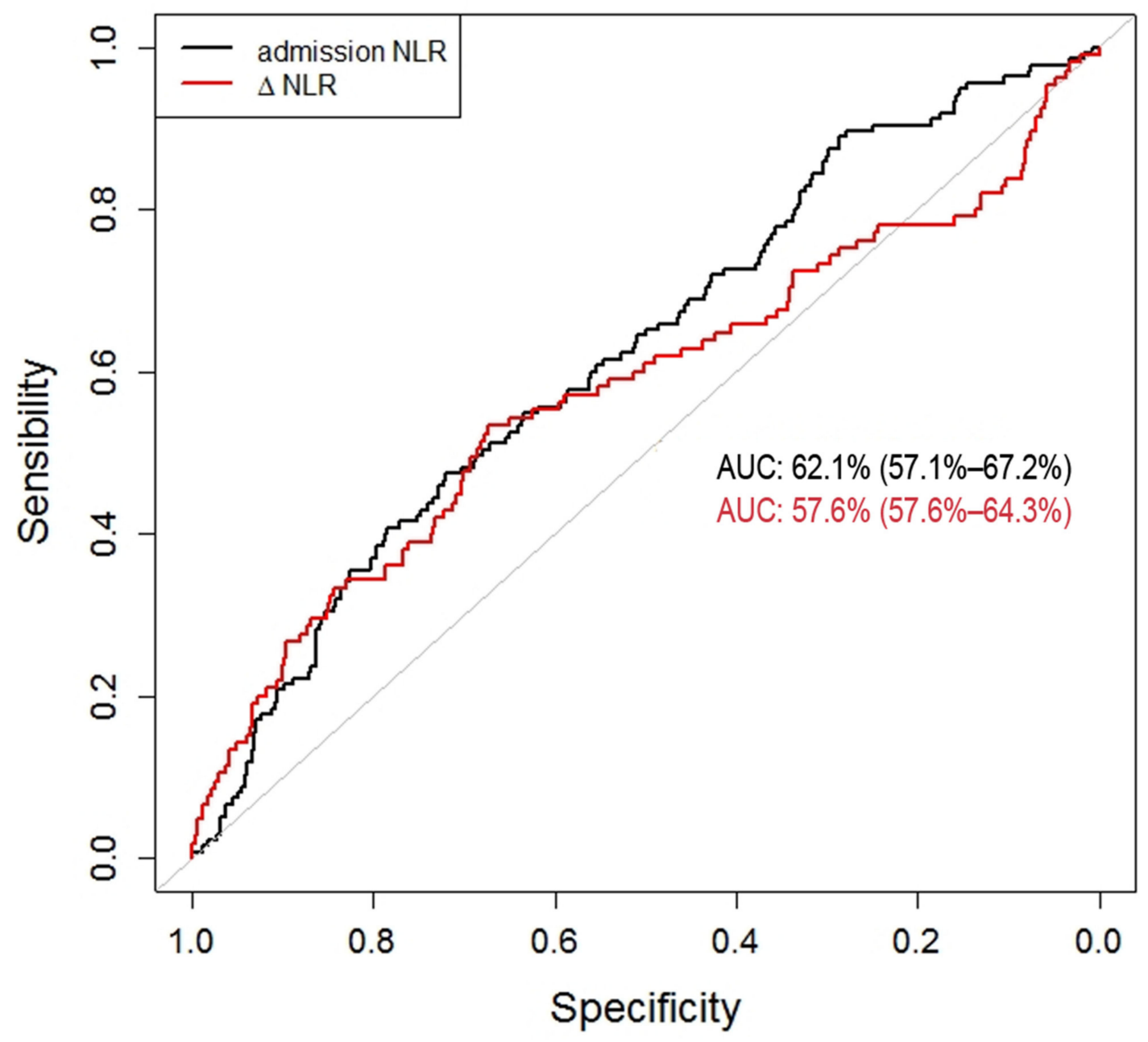

\begin{tabular}{ccccccc}
\hline \multirow{2}{*}{$\begin{array}{c}\text { To Predict } \\
\text { Mortality }\end{array}$} & Area under Curve & Cut-Off & Sensibility & Specificity & \multicolumn{2}{c}{$\begin{array}{c}\text { Multivariate Analysis with Cut- } \\
\text { Off ** }\end{array}$} \\
\cline { 5 - 7 } & & & & & OR (95\%CI) & $p$ \\
\hline Admission NLR & $0.621(0.571-0.672)$ & 8.236 & $47.4(38.8-56.2)$ & $71.9(68.8-74.9)$ & $1.025(0.989-1.063)$ & 0.176 \\
$\Delta N L R$ & $0.576(0.509-0.643)$ & 0.411 & $53.3(43.3-63.1)$ & $67.3(63.7-70.8)$ & $2.71(1.404-5.245)$ & $0.003 *$ \\
\hline
\end{tabular}

Data are expressed in median (Q1-Q3), ${ }^{*} p<0.005,{ }^{* *}$ model adjusted for Gender, Body mass index, C reactive protein, Creatinine, Admission NLR, NLR H24 and $\triangle$ NLR.

Abbreviations: OR = odds ratio, NLR = neutrophil lymphocyte ratio, $\triangle N L R=$ difference between NLR (NLR H-24-NLR at admission).

Figure 3. Receiver operating characteristics (ROC) curve for NLR as a predictive factor of mortality in COVID-19 patients. 


\subsection{Validation Cohort}

The AUC of the training and validation cohorts were respectively 0.598 and 0.591 to predict critical illness and 0.629 and 0.616 to predict intra-hospital mortality, which validates the models. All data regarding the validation and training cohorts are available (Figures S1 and S2).

\section{Discussion}

The main endpoint of our study was to investigate the prognostic value of NLR in a cohort of patients infected with SARS-CoV-2, upon their admission to the ED and in the first $24 \mathrm{~h}$ of management $(\mathrm{H}-24)$. We selected patients as rigorously as possible to minimize all confounding factors that could alter the WBC. Our study confirmed that NLR is a potential marker to discriminate severity and mortality in patients with SARS-CoV-2 infection. In addition, we demonstrated the relevance of using $\triangle N L R$ to predict severity and mortality regarding this emerging disease.

Our results reflect other studies on pulmonary infections, where the NLR level upon admission to the ED was predictive of severity and mortality more accurately than other conventional biomarkers such as CRP [5,6]. De Jager et al. [5] established, in a cohort of 395 patients, an AUC of 0.701 for NLR, compared to 0.565 for CRP, 0.681 for neutrophils alone, and 0.672 for lymphocytes alone. In a prospective clinical study on 195 elderly subjects with acute pulmonary infection, Cataudella et al. [18] reported that NLR was an excellent predictor of mortality at 30 days, along with a correlation between the level of NLR and mortality (50\% mortality for 13.4-28.3 NLR and 100\% mortality if higher than 28.3 NLR). Concerning SARS-CoV-2 infection, NLR was also independently associated with progression to critical illness [19-22]. This association was demonstrated by Lian and al. [19] who also reported that the relationship between NLR and disease progression was significant and graded (HR:1.16 (CI 95\%: $(1.10-1.22) ; p<0.001)$ ). Moreover, NLR appears to be a predictive marker of mortality in this infection, as found in the meta-analysis of $\mathrm{Li}$ and al. [17], which included 10 studies and 2967 patients with sensitivity, specificity, and AUC values of $0.83,0.83$, and 0.90 respectively. These studies, although highlighting the prognostic value of NLR, did not take into account certain patient-related parameters potentially affecting the lymphocyte formula and, de facto, the NLR, nor did they precisely define the time of NLR measurement and its variation. Conclusively, our results stand more explicit: we refined our cohort by excluding patients who might have other causes modifying their lymphocyte formula and we clearly defined NLR measurements in detail. Therefore, our findings bring a novel and potential better view over the effect of SARSCoV-2 on white blood cells (WBC) count.

Accordingly, there is great interest in considering NLR for its prognostic value in SARSCoV-2 infection. Regarding clinical severity, we were able to demonstrate a significant difference in NLR upon admission to the ED when comparing moderate and severe infections. The difference between the two was even more relevant at $\mathrm{H}-24$, which brings forward a marker that is specifically and significantly associated with severity and mortality, i.e., $\triangle N L R$, which is the difference between NLR values at $\mathrm{H}-24$ and upon admission to the ED. In our cohort, positive $\triangle N L R$, signifying an increase in NLR between admission and H-24, was associated with a poor prognosis. Ye et al. [9] found similar results for a cohort of 349 patients, with an increase in NLR during hospitalization significantly associated with mortality. Riché et al. observed a reversed correlation in patients with septic shock [23]. Studies have indicated various thresholds to NLR [17] and, similarly to our findings, an NLR median value of 4.5 is often described in severe SARS-CoV-2 infections, with a recently demonstrated specificity of $86 \%$ and sensitivity of $74 \%$ [24]. On the other hand, in a population of 12,862 COVID-19 patients, Cai et al. [25] described an NLR threshold of 6.11 , with sensitivity and specificity values of, respectively, $76 \%$ and $87 \%$ for predicting mortality, hence implying that, above this threshold, the introduction of corticosteroid therapy significantly reduced mortality. 
One of the major keys in the management of this outbreak is controlling the number of patients in the ICU, which is the main reason behind the oversaturation of healthcare systems, an overload that increases mortality [26]. Therefore, the orientation of a COVID-19 patient between a conventional hospitalization unit or the ICU is essential. The value of the NLR could be coupled with that of other markers in a multi-marker approach or in a combined severity prediction score; this might be the key allowing optimization and standardization of patient triage in the ED.

\section{Limitations}

Our study presents several limitations. First, it is retrospective, which means that, although we added a number of exclusion criteria (notably, comorbidities modifying the blood count and, therefore, the circulating lymphocyte and neutrophil count), the data are subject to other confounding factors. These data were collected during first wave of the pandemic, and at that time, the vast majority of our patients did not receive corticosteroid treatments modifying their WBC count, which are nowadays one of the cornerstones of severe SARS-CoV-2 infection management. Similarly, many patients were admitted to the ED after receiving non-recommended antibiotics that also affect the WBC count; these patients were also excluded from the study. Second, the $\triangle N L R$ may have been influenced by other factors related to patient management that we did not consider in the analysis, for lack of information that could not be exhaustively detailed. Decisively, the retrospective nature of the study did not allow us to extensively control the existence of other confounding factors such as bacterial co-infections, which could have allowed us to further refine our results.

\section{Conclusions}

The NLR and its early variation $(\triangle N L R)$ could help physicians predict both severity and mortality associated with SARS-CoV-2 infection, hence contributing to optimized patient management (accurate triage and treatment).

Supplementary Materials: The following are available online at https: / www.mdpi.com/article / 10.3390/jcm10122563/s1, Table S1: First apprentissage-validation: demographic and baseline characteristics of patients with COVID-19, Table S2: First apprentissage-validation: laboratory findings of patients with COVID-19. Table S3: Second apprentissage-validation: demographic and baseline characteristics of patients with COVID-19, Table S4: Second apprentissage-validation: laboratory findings of patients with COVID-19, Figure S1: Receiver operating characteristics curve for ability of NLR to predict critical illness, Figure S2: Receiver operating characteristics curve for ability of NLR to predict in-hospital mortality.

Author Contributions: L.A.V., P.L.B., and C.-E.L. conceived the presented idea. P.L.B., L.A.V., P.B., and C.-E.L. developed the theory and performed the computations. L.A.V., P.L.B., F.L., and C.-E.L. verified the analytical methods. All authors collected data, discussed the results, and contributed to the final manuscript. All authors have read and agreed to the published version of the manuscript.

Funding: This research received no external funding.

Institutional Review Board Statement: This study was approved by the institution's (Strasbourg University Hospital, France) Ethics Review Board (reference: CE-2020-39).

Informed Consent Statement: Patient consent was waived, in accordance with the French legislation, waived the need for informed consent of patients whose data were entirely retrospectively studied.

Data Availability Statement: All data analyzed as part of the study are included.

Acknowledgments: All authors would especially like to thank all hospital staff, physicians, nurses, and all healthcare workers who faced this pandemic.

Conflicts of Interest: All authors declare no conflict of interest. 


\section{References}

1. Zhu, N.; Zhang, D.; Wang, W.; Li, X.; Yang, B.; Song, J.; Zhao, X.; Huang, B.; Shi, W.; Lu, R.; et al. A Novel Coronavirus from Patients with Pneumonia in China, 2019. N. Engl. J. Med. 2020, 382, 727-733. [CrossRef]

2. Qin, C.; Zhou, L.; Hu, Z.; Zhang, S.; Yang, S.; Tao, Y.; Xie, C.; Ma, K.; Shang, K.; Wang, W.; et al. Dysregulation of immune response in patients with COVID-19 in Wuhan China. Clin. Infect. Dis. 2020, 75, 762-768. [CrossRef]

3. Fajgenbaum, D.C.; June, C.H. Cytokine Storm. N. Engl. J. Med. 2020, 383, 2255-2273. [CrossRef] [PubMed]

4. Pitre, T.; Jones, A.; Su, J.; Helmeczi, W.; Xu, G.; Lee, C.; Shamsuddin, A.; Mir, A.; MacGregor, S.; Duong, M.; et al. Inflammatory biomarkers as independent prognosticators of 28-day mortality for COVID-19 patients admitted to general medicine or ICU wards: A retrospective cohort study. Intern. Emerg. Med. 2021, 26, 1-10. [CrossRef]

5. De Jager, C.P.; Wever, P.C.; Gemen, E.F.; Kusters, R.; van Gageldonk-Lafeber, A.B.; van der Poll, T.; Laheij, R.J. The neutrophillymphocyte count ratio in patients with community-acquired pneumonia. PLoS ONE 2012, 7, e46561. [CrossRef]

6. Paliogiannis, P.; Fois, A.G.; Sotgia, S.; Mangoni, A.A.; Zinellu, E.; Pirina, P.; Negri, S.; Carru, C.; Zinellu, A. Neutrophil to lymphocyte ratio and clinical outcomes in COPD: Recent evidence and future perspectives. Eur. Respir. Rev. 2018, $27,170113$.

7. Chan, A.S.; Rout, A. Use of Neutrophil-to-Lymphocyte and Platelet-to-Lymphocyte Ratios in COVID-19. J. Clin. Med. Res. 2020, 12, 448-453. [CrossRef] [PubMed]

8. Rosales, C. Neutrophil: A Cell with Many Roles in Inflammation or Several Cell Types? Front. Physiol. 2018, 9, 113. [CrossRef]

9. Laforge, M.; Elbim, C.; Frère, C.; Hémadi, M.; Massaad, C.; Nuss, P.; Benoliel, J.J.; Becker, C. Tissue damage from neutrophilinduced oxidative stress in COVID-19. Nat. Rev. Immunol. 2020, 20, 515-516. [CrossRef] [PubMed]

10. Guthrie, G.J.; Charles, K.A.; Roxburgh, C.S.; Horgan, P.G.; McMillan, D.C.; Clarke, S.J. The systemic inflammation-based neutrophil-lymphocyte ratio: Experience in patients with cancer. Crit. Rev. Oncol. Hematol. 2013, 88, 218-230. [CrossRef]

11. Huang, Z.; Fu, Z.; Huang, W.; Huang, K. Prognostic value of neutrophil-to-lymphocyte ratio in sepsis: A meta-analysis. Am. J. Emerg. Med. 2020, 38, 641-647. [CrossRef] [PubMed]

12. Hwang, S.Y.; Shin, T.G.; Jo, I.J.; Jeon, K.; Suh, G.Y.; Lee, T.R.; Yoon, H.; Cha, W.C.; Sim, M.S. Neutrophil-to-lymphocyte ratio as a prognostic marker in critically-ill septic patients. Am. J. Emerg. Med. 2017, 35, 234-239. [CrossRef]

13. Templeton, A.J.; McNamara, M.G.; Šeruga, B.; Vera-Badillo, F.E.; Aneja, P.; Ocaña, A.; Leibowitz-Amit, R.; Sonpavde, G.; Knox, J.J.; Tran, B.; et al. Prognostic role of neutrophil-to-lymphocyte ratio in solid tumors: A systematic review and meta-analysis. J. Natl. Cancer Inst. 2014, 106, dju124. [CrossRef]

14. De Jager, C.P.; Vvan Wijk, P.T.; Mathoera, R.B.; de Jongh-Leuvenink, J.; van der Poll, T.; Wever, P.C. Lymphocytopenia and neutrophil-lymphocyte count ratio predict bacteremia better than conventional infection markers in an emergency care unit. Crit. Care 2010, 14, R192. [CrossRef] [PubMed]

15. Kong, M.; Zhang, H.; Cao, X.; Mao, X.; Lu, Z. Higher level of neutrophil-to-lymphocyte is associated with severe COVID-19. Epidemiol. Infect. 2020, 148, e139. [CrossRef]

16. Fu, J.; Kong, J.; Wang, W.; Wu, M.; Yao, L.; Wang, Z.; Jin, J.; Wu, D.; Yu, X. The clinical implication of dynamic neutrophil to lymphocyte ratio and D-dimer in COVID-19: A retrospective study in Suzhou China. Thromb. Res. 2020, 192, 3-8. [CrossRef]

17. Li, X.; Liu, C.; Mao, Z.; Xiao, M.; Wang, L.; Qi, S.; Zhou, F. Predictive values of neutrophil-to-lymphocyte ratio on disease severity and mortality in COVID-19 patients: A systematic review and meta-analysis. Crit. Care 2020, 24, 647. [CrossRef]

18. Cataudella, E.; Giraffa, C.M.; Di Marca, S.; Pulvirenti, A.; Alaimo, S.; Pisano, M.; Terranova, V.; Corriere, T.; Ronsisvalle, M.L.; Di Quattro, R.; et al. Neutrophil-To-Lymphocyte Ratio: An Emerging Marker Predicting Prognosis in Elderly Adults with Community-Acquired Pneumonia. J. Am. Geriatr. Soc. 2017, 65, 1796-1801. [CrossRef] [PubMed]

19. Lian, J.; Jin, C.; Hao, S.; Zhang, X.; Yang, M.; Jin, X.; Lu, Y.; Hu, J.; Zhang, S.; Zheng, L.; et al. High neutrophil-to-lymphocyte ratio associated with progression to critical illness in older patients with COVID-19: A multicenter retrospective study. Aging 2020, 12, 13849-13859. [CrossRef] [PubMed]

20. Salciccioli, J.D.; Marshall, D.C.; Pimentel, M.A.; Santos, M.D.; Pollard, T.; Celi, L.A.; Shalhoub, J. The association between the neutrophil-to-lymphocyte ratio and mortality in critical illness: An observational cohort study. Crit. Care 2015, 19, 13. [CrossRef]

21. Liu, Y.P.; Li, G.M.; He, J.; Liu, Y.; Li, M.; Zhang, R.; Li, Y.L.; Wu, Y.Z.; Diao, B. Combined use of the neutrophil-to-lymphocyte ratio and CRP to predict 7-day disease severity in 84 hospitalized patients with COVID-19 pneumonia: A retrospective cohort study. Ann. Transl. Med. 2020, 8, 635. [CrossRef] [PubMed]

22. Yang, A.P.; Liu, J.P.; Tao, W.Q.; Li, H.M. The diagnostic and predictive role of NLR, d-NLR and PLR in COVID-19 patients. Int. Immunopharmacol. 2020, 84, 106504. [CrossRef] [PubMed]

23. Riche, F.; Gayat, E.; Barthelemy, R.; Le Dorze, M.; Mateo, J.; Payen, D. Reversal of neutrophil-to-lymphocyte count ratio in early versus late death from septic shock. Crit. Care 2015, 19, 439. [CrossRef] [PubMed]

24. Ciccullo, A.; Borghetti, A.; Dal Verme, L.Z.; Tosoni, A.; Lombardi, F.; Garcovich, M.; Biscetti, F.; Montalto, M.; Cauda, R.; Di Giambenedetto, S.; et al. Neutrophil-to-lymphocyte ratio and clinical outcome in COVID-19: A report from the Italian front line. Int. J. Antimicrob. Agents 2020, 56, 106017. [CrossRef] [PubMed]

25. Cai, J.; Li, H.; Zhang, C.; Chen, Z.; Liu, H.; Lei, F.; Qin, J.J.; Liu, Y.M.; Zhou, F.; Song, X.; et al. The Neutrophil-to-Lymphocyte Ratio Determines Clinical Efficacy of Corticosteroid Therapy in Patients with COVID-19. Cell Metab. 2021, 33, 258-269.e3. [CrossRef]

26. Bravata, D.M.; Perkins, A.J.; Myers, L.J.; Arling, G.; Zhang, Y.; Zillich, A.J.; Reese, L.; Dysangco, A.; Agarwal, R.; Myers, J.; et al. Association of Intensive Care Unit Patient Load and Demand with Mortality Rates in US Department of Veterans Affairs Hospitals During the COVID-19 Pandemic. JAMA Netw. Open 2021, 4, e2034266. [CrossRef] [PubMed] 